\title{
La UCLAEH en tiempos de pandemia
}

La que sigue es una breve reseña de las diversas iniciativas y acciones que la UCLAEH ha llevado a cabo mediante sus distintos programas y facultades en el contexto de la pandemia de la COVID-19.

La pandemia y sus consecuencias han configurado una situación de insólita complejidad, cuya secuela no podemos adelantar con detalle, frente a la cual tal vez la opción más prudente sería callar y esperar: la lechuza de Minerva solo emprende su vuelo a la caída de la noche. Pero - al decir de Ortega y Gasset—, sin perjuicio de su misión científica, «la Universidad tiene que estar también abierta a la plena actualidad; más aún: tiene que estar en medio de ella, sumergida en ella». Y la UCLAEH no se caracteriza por esperar. Sabe desde su propia médula que la investigación y el análisis tienen vocación de insertarse en la realidad, contiene en su misma configuración genética el impulso de leer la realidad y transformarla.

Por decreto 93/2020, del 13 de marzo de 2020, el Poder Ejecutivo declaró el estado de emergencia nacional sanitaria. A su vez, el decreto 101/2020, del 16 de marzo de 2020, dispuso, con relación a los centros privados de enseñanza de todos los niveles, la «suspensión del dictado de clases y el cierre de los centros educativos». El 16 de marzo de 2020, el Consejo Superior de la Universidad discutía las acciones de la Universidad ante la pandemia del COVID-19 y la situación provocada, y comunicaba el Plan de Contingencia COVID-19 de la Universidad CLAEH para reordenar la actividad de docentes, estudiantes y funcionarios. Podemos ver en retrospectiva la gran incertidumbre que se había instalado, ante la cual primero sobrevino la improvisación, pero enseguida aparecieron enormes posibilidades en la tecnología: las plataformas Zoom y Moodle, ya usadas, aunque en escala menor, permitieron retomar la actividad y seguir caminando.

Por otra parte, todas las unidades académicas de la Universidad y sus programas de intervención social se abocaron a encontrar, desde sus respectivas disciplinas, información confiable y respuestas a las innumerables preguntas que se sucedían, haciendo un gran esfuerzo por dar un mensaje coherente frente al bombardeo de información.

Damos cuenta sucinta de esas actividades llevadas a cabo desde el 16 de marzo de 2020 en las distintas unidades académicas. 


\section{Facultad de Medicina}

En la Facultad de Medicina, bajo la dirección de la decana interina, Dra. Selva Lima, inmediatamente todos los docentes continuaron su actividad utilizando las herramientas disponibles: plataformas Moodle y Zoom, entre otras. Con la visión que permite la retrospectiva, se puede concluir que, si bien fue un gran desafío, tanto docentes como alumnos se fueron adaptando a las nuevas modalidades educativas. Los cursos se han cumplido casi en su totalidad; solo queda insatisfecha la necesidad de la asistencia a la práctica clínica, que se reiniciará en el momento oportuno.

Concomitantemente, para aportar en la lucha contra la pandemia, docentes y alumnos trabajaron en el diseño de un cronograma sintomático para realizar mediante interrogatorio telefónico (triaje telefónico), a solicitud de los centros asistenciales, ante la posibilidad de un desborde asistencial. Para este triaje, que permitiría ubicar pacientes sospechosos de COVID-19, valorar la necesidad de asistencia domiciliaria o de acudir al centro asistencial, se apeló a voluntariado entre alumnos a partir de cuarto año. En la instrucción, los alumnos trabajaron junto con los docentes.

Otra actividad extracurricular realizada por alumnos de diferentes años fue la divulgación de información acerca de elementos de alarma y medidas de cuidados entre la población.

Se diseñaron proyectos de investigación vinculados al proceso COVID-19, que están en consideración en el Comité de Ética.

Especial relevancia tiene el servicio de información científica a través del blog https://www.humamed.info/, que se actualiza diariamente, disponible en la página de la UCLAEH, acerca de lo publicado en revistas de relevancia académica de todo el mundo sobre el coronavirus, día por día desde el 16 de marzo de 2020, que permite estar al tanto de las últimas investigaciones sobre los diferentes aspectos de la pandemia.

La información que la Facultad divulga proviene de artículos científicos rigurosos y se publica con la fuente que corresponde. El trabajo de selección es responsabilidad del decano emérito Prof. Dr. Humberto Correa y del docente de Investigación y Medicina basada en evidencia Dr. Edgardo Sandoya.

Los artículos se publican traducidos en el blog, que se creó especialmente. Con esta iniciativa, la Facultad aporta información de calidad como elemento indispensable en la situación de emergencia sanitaria.

Se elaboraron también publicaciones sobre diferentes impactos de la COVID-19, en las que participaron docentes de diferentes áreas.

La Facultad fue convocada para colaborar en una actividad de alcance nacional realizada el 27 de abril de 2020, coordinada por el decano de la Facultad de Ciencias de la Salud de la Universidad Católica, dirigida al cuerpo de enfermería de Hospital Español, 
institución nacional de referencia en materia de cuidados en tiempos de COVID-19. La Facultad colaboró con la participación de docentes referentes.

De igual forma, la Facultad participó a través de sus docentes en actividades coordinadas por el Colegio Médico del Uruguay, y realizará para esta organización un curso de Medicina Basada en la Evidencia.

Se planificaron y están llevando a cabo varios protocolos de investigación desarrollados por los alumnos con la supervisión del docente a cargo del Programa de Medicina Basada en Evidencia e Investigación.

Se desarrollaron actividades mediante plataformas en línea, organizadas por grupos de docentes, con participación activa de los alumnos de los últimos años de la carrera, acerca de diferentes temas de interés médico.

Se conformaron asimismo grupos de docentes abocados a mejorar académicamente la metodología educativa, a planificar nuevos aspectos para integrar en la carrera de grado y posgrado, a planificar la integración de la Facultad en otras áreas, así como su proyección nacional e internacional.

En marzo de 2020, 21 nuevos graduados de la Facultad de Medicina obtuvieron el registro de sus títulos en tiempo récord, superando toda dificultad burocrática, con lo que quedaron habilitados para colaborar desde su profesión en el combate contra la pandemia.

\section{Facultad de Derecho}

Ante la suspensión de la actividad presencial, la Facultad de Derecho se abocó inmediatamente a la tarea de generar las condiciones que permitieran darle continuidad al desarrollo de los cursos, mediante el empleo de todas las herramientas que se tenían al alcance. Gracias al trabajo del equipo de dirección, compuesto por el decano, Dr. Mario Garmendia, y la secretaria académica, Dra. Gabriela Ipharraguerre, en un fin de semana (14 y 15 de marzo) se cargaron en la plataforma Moodle todos los cursos de las dos carreras que se dictan en la Facultad. Eso posibilitó que docentes y estudiantes quedaran en condiciones de continuar los cursos de forma remota el lunes 16 de marzo, es decir, sin pérdida de días de clase. También fue posible dar la bienvenida a la generación de ingreso (2020), mediante un mensaje audiovisual del suscrito, y comenzar los cursos, tal como estaba previsto originalmente, el 23 de marzo. No bien estuvo disponible la plataforma Zoom, se realizó un intenso trabajo de capacitación de los docentes, mediante tutoriales y contactos personalizados. Todo esto permite que, al día de la fecha, podamos decir que todos los cursos de la Facultad de Derecho pudieron seguir desarrollándose sin solución de continuidad. 
Cabe puntualizar que, en atención a que los cursos de la Facultad incluyen la exigencia de una asistencia mínima obligatoria, se instruyó a los docentes para que controlaran y registraran la asistencia de los estudiantes y que les solicitaran mantener sus cámaras encendidas durante el desarrollo de las clases, a fin de incentivar su participación oral y promover su calificación, según los criterios habituales que aplica el docente. Estas directivas fueron cumplidas en forma satisfactoria, a pesar de ciertos inconvenientes - no significativos - que tuvieron algunos estudiantes con sus conexiones a internet.

También se adoptaron medidas dirigidas a brindar las garantías para la realización de evaluaciones, pruebas y exámenes. La Vicerrectoría Académica elaboró un documento que sintetiza los lineamientos generales a aplicar en materia de evaluaciones, que podían adaptarse a las características de cada facultad y a las particularidades de cada asignatura y curso. En la Facultad de Derecho se dispuso que cada docente definiría y comunicaría claramente a los estudiantes, con razonable anticipación, la modalidad de evaluación que aplicaría en su curso. Se indicó a los docentes que el método debía elegirse teniendo en cuenta los objetivos que se persiguen con la realización de las pruebas y procurando que la modalidad escogida brindara garantías razonables en materia de autenticidad, autoría, transparencia, igualdad de oportunidades, etc. A modo de ejemplo, se indicó que el docente podría proponer trabajos externos individuales o grupales, participación en foros, pruebas de conocimiento escritas u orales, con hora de inicio y finalización (con la opción de utilizar Zoom), que se entregarían vía email, plataforma Moodle o similar, etc. Se destacó la especial importancia de que en la formulación de las pruebas se priorizara la evaluación del proceso de razonamiento y aplicación práctica de los conocimientos por parte del estudiante.

Este importante esfuerzo docente fue acompañado, igual que en la Facultad de Medicina, por otras actividades académicas que contribuyeron al enfoque de los problemas jurídicos generados por la pandemia desde el punto de vista académico.

\section{Seminario Virtual: COVID-19 y políticas laborales: análisis desde una perspectiva comparada}

La Facultad coorganizó con la Pontificia Universidad Católica de Valparaíso (Chile) un seminario virtual sobre el tema «Respuestas del derecho del trabajo y de la seguridad social ante la pandemia. Panorama comparado». Este se llevó a cabo en dos jornadas (miércoles 15 y jueves 16 de abril de 2020) y contó con la participación de expositores de Argentina, Brasil, Colombia, Chile, España, México, Paraguay, Perú y Uruguay.

La actividad se desarrolló mediante Zoom y fue transmitida a través de YouTube, con una audiencia que por momentos superó las 500 personas. 


\section{Conferencia «La función notarial en tiempos de pandemia»}

Esta conferencia, a cargo de la profesora argentina Rosario Stoppani, tuvo lugar el 29 de abril de 2020 mediante la plataforma Zoom.

\section{Conferencia «La imprevisión en países que no la regulan. Desa- fío jurisprudencial»}

Dictada por el vicerrector académico de la Universidad, Dr. Carlos de Cores, el 28 de abril de 2020, esta conferencia virtual fue organizada por la Asociación Iberoamericana de Derecho Privado.

\section{Producción doctrinaria, material de difusión e información}

El decano exhortó a los docentes a que realizaran trabajos académicos que tuvieran relación con la emergencia sanitaria nacional. Los numerosos y sustanciales aportes (documentos escritos y videos) se encuentran accesibles en el sitio web de la Facultad de Derecho (http://universidad.claeh.edu.uy/derecho/) y sus autores son los siguientes: derecho del trabajo y seguridad social, Dr. Álvaro Rodríguez Azcúe y Dr. Mario Garmendia Arigón; derecho penal, Dr. Gustavo Bordes; derecho constitucional, Dr. Jaime Sapolinski; derecho administrativo, Prof. Graciela Fernández.

\section{Facultad de la Cultura}

La actividad docente de la Facultad de la Cultura continuó en toda su extensión por medio de las plataformas Moodle y Zoom bajo la dirección de su decano, Mag. Javier Dotta, y del secretario académico, Danilo Urbanavicius.

Con la participación de docentes de la Facultad y de docentes y referentes de la cultura invitados, uruguayos y extranjeros, se realizaron dos foros abiertos:

El del miércoles 29 de abril tuvo por título «El rol del gestor cultural en la pandemia». Moderado por Gerardo Grieco, contó la participación de Ana Agostino, Roberto Elissalde, Paula Delgado, Belén Díaz, Andrea Fantoni, Diego Gómez, Erika Hoffman, Luis Mardones, Fernando Ordóñez y Mariné Villalba, entre otros.

Cómo será la ciudad? ¿Cómo serán los teatros? ¿Qué harán las y los artistas? ¿Cómo cambiarán los públicos? Estas son algunas de las preguntas con las que Gerardo Grieco guio la reflexión y el análisis.

Esta iniciativa de la Facultad de la Cultura de la Universidad CLAEH intentó levantar la mirada y aportar una perspectiva sobre la responsabilidad de los gestores culturales en la salida de la crisis del coronavirus. Para Grieco - docente y gestor cultural de referencia-, la idea del encuentro fue «pensar qué tenemos para aportar, cuál es el rol 
de la gestión cultural en este momento», una perspectiva propositiva alejada de los reclamos de medidas o presupuesto. El foro contó con más de 200 asistentes.

Por otra parte, el viernes 22 de mayo, en el marco de la Especialización en Historia del Arte y Patrimonio, se dictó la clase abierta «Civilizar la pandemia. Una mirada al arte en tiempos de COVID-19», a cargo de Carolina Porley.

Una contribución destacada de la Facultad al análisis del impacto de la pandemia y de los decretos gubernamentales que suspendieron las diversas actividades, entre ellas los espectáculos públicos (artículo 3 del decreto del Poder Ejecutivo 93/2020, de 13 de marzo de 2020), fue el artículo titulado «Estimación del impacto de la pandemia en las artes escénicas en Uruguay», de Ernesto Pienika y Diego Traverso, disponible en http://universidad.claeh.edu.uy/cultura/wp-content/uploads/sites/6/2020/04/Art_FacultaddelaCultura_202004.pdf

El texto se enmarca en el estudio de la Cuenta Satélite de la Cultura del Uruguay (CSCU) que viene realizando la Facultad de la Cultura de la Universidad CLAEH. Esta investigación mide económicamente a los sectores culturales y el impacto que tienen en el PIB nacional. El proyecto consiste en darle continuidad a la medición económica de la cultura realizada en las CSCU en 2009 y 2012, revisando la metodología y tomando el año 2016 como referencia de análisis.

En esa primera instancia los sectores relevados fueron artes escénicas y música (producción de fonogramas, tanto físicos como digitales, y espectáculos en vivo).

Con algunos datos ya sistematizados para la Cuenta Satélite de Cultura en Uruguay y el análisis del comportamiento estacional de los distintos sectores, se estimó la magnitud del impacto de la pandemia del coronavirus en el área de las artes escénicas en el país, con previsión de impactos de significativa magnitud aun en el escenario más optimista, de que los espectáculos públicos se reanudaran el $1 .^{\circ}$ de junio.

Asimismo, cabe reseñar el artículo «El sector cultural del Uruguay en tiempos de pandemia», de Danilo Urbanavicius, secretario académico de la Facultad de la Cultura de la Universidad CLAEH, disponible en http://rgcediciones.com.ar/el-sector-culturaldel-uruguay-en-tiempos-de-pandemia/

Los referentes de la Facultad han realizado también distintas contribuciones en prensa oral y escrita:

- «La crisis y el sector cultural: investigadores del CLAEH estiman impactos y posibles escenarios», en La Diaria, disponible en https://ladiaria.com.uy/articulo/2020 /4/la-crisis-y-el-sector-cultural-investigadores-del-claeh-estiman-impactos-y-posibles-escenarios/

- «El impacto de la pandemia en las artes escénicas», entrevista con Diego Traverso en Radio Sarandí, disponible en https://www.sarandi690.com.uy/2020/04/20/elimpacto-de-la-pandemia-en-las-artes-escenicas / 
- «Convocatoria al foro virtual sobre el rol de la gestión cultural en la pandemia», Javier Dotta en Radio Sarandí, disponible en https://www.sarandi690.com.uy/2020/ 04/28/convocan-a-foro-virtual-sobre-el-rol-de-la-gestion-cultural-en-la-pandemia/

\section{Programa de Desarrollo Local}

La Universidad CLAEH, con el grupo motor de la Red de Desarrollo Económico Territorial (RED DET, Uruguay) y ANDE, coorganizaron el conversatorio «COVID-19: aportes desde el territorio». Este tuvo lugar el miércoles 22 de abril de 2020, con el propósito de generar un diálogo entre territorios sobre abordajes, prácticas y factores de desarrollo económico territorial ante la emergencia sanitaria y frente a escenarios de salida. El conversatorio se enmarcó en las actividades «En diálogo», dirigidas a la profundización de conocimientos y el intercambio entre distintos agentes de desarrollo vinculados al desarrollo territorial.

Por otra parte, siempre en el marco de la Red de Desarrollo Económico Territorial, debe anotarse que se encuentra en curso una encuesta en la que se invita a compartir experiencias y respuestas locales en relación con la economía local ante la emergencia sanitaria.

El propósito es visibilizar las experiencias e iniciativas locales en esta temática, compartirlas y sistematizarlas para favorecer el aprendizaje colectivo de abordajes, prácticas y factores de desarrollo económico territorial ante la coyuntura y los escenarios emergentes, para hacer posible circular la información y compartir la diversidad de acciones y factores clave en los distintos territorios en relación con los temas que involucran al desarrollo económico local.

\section{CLAEH en Línea}

Un lugar destacado, por supuesto, corresponde al equipo que gestiona la tecnología que hace posible la enseñanza en línea. La intensa actividad que llevaba a cabo la Universidad antes de la pandemia en el marco de su campus virtual se multiplicó.

Además de múltiples cursos de apoyo a docentes para el uso de la plataforma Moodle, el equipo de CLAEH en Línea está llevando a cabo el curso «Educar en línea», bajo la dirección de Ricardo Garay, con el objetivo de analizar la propia práctica docente, proyectarla hacia ámbitos virtuales, imaginar nuevos diseños educativos e ir construyendo, colaborativamente, una praxis educativa para este tiempo.

El curso abarca en esta etapa cuatro temas: Educación e internet, Redes en las aulas, Diseño de cursos en espacios virtuales y Creación de actividades educativas en línea. 


\section{Programa de Educación}

El 18 de mayo en el programa Todas las voces, el titular del Programa de Educación, Prof. Pablo Cayota, junto con el Dr. Renato Opertti, participaron en un especial titulado «Cómo educar después de la pandemia». Se aportaron sólidas conclusiones sobre lo que ha llegado para quedarse: la incorporación de tecnología, que se revela como un componente esencial de la actividad educativa en el siglo XXI.

No obstante, quedó claro que la virtualidad no puede operar como alternativa, sino como complemento de la educación presencial, un requisito imprescindible que debe recuperarse tan pronto sea posible.

\section{Programa de Salud}

Se ha realizado una adaptación de los contenidos de las diferentes asignaturas de la especialización, tanto en primero como en segundo año, así como también en los programas de maestría.

Por otra parte, el Programa está en condiciones de definir el lanzamiento de un nuevo curso, Epidemiología de las Enfermedades Transmisibles, muy adaptado a las actuales necesidades de formación de los profesionales que trabajan en el sector.

\section{Conclusión}

La magnitud de los cambios que estamos viviendo en este tiempo elimina toda posibilidad de formular conclusiones exhaustivas.

Sin embargo, desde el ADN de la Universidad CLAEH, desde su misión y visión, luce claro que esta pandemia de la COVID-19 ha desnudado vulnerabilidades e inequidades de los sistemas sociales, económicos y culturales que no son aceptables en el estado actual de la conciencia colectiva.

Pero al mismo tiempo está señalando orientaciones precisas para dar un paso adelante en la historia, en el sentido de la utopía que se puede entrever a partir de trazos borrosos e imprecisos. No para construir la sociedad definitiva, el paraíso terrenal, fuente histórica de importantes regresos, sino para definir mejoras efectivas en el sentido del desarrollo de todos los hombres y de todo el hombre, que constituye el objeto de la economía humana.

Todas las fuerzas de la comunidad universitaria de la UCLAEH asumen en esta coyuntura, más que nunca, el compromiso de extremar la inteligencia para ir definiendo 
herramientas conceptuales que permitan entender y definir con solidez las mejoras posibles, y habilitar así avances efectivos de las poblaciones, de condiciones menos humanas a condiciones más humanas.

Carlos de Cores

Vicerrector académico

Universidad CLAEH 